\title{
ARTICLE OPEN \\ Polymeric foams for flexible and highly sensitive low-pressure capacitive sensors
}

\author{
Mickaël Pruvost ${ }^{1}{ }^{1}$, Wilbert J. Smit ${ }^{1}$, Cécile Monteux ${ }^{2}$, Philippe Poulin ${ }^{3}$ and Annie Colin ${ }^{1}$
}

Flexible low-pressure sensors ( $<10 \mathrm{kPa}$ ) are required in areas as diverse as blood-pressure monitoring, human-computer interactions, robotics, and object detection. For applications, it is essential that these sensors combine flexibility, high sensitivity, robustness, and low production costs. Previous works involve surface micro-patterning, electronic amplification (OFET), and hydrogels. However, these solutions are limited as they involve complex processes, large bias voltages, large energy consumption, or are sensitive to evaporation. Here, we report a major advance to solve the challenge of scalable, efficient and robust e-skin. We present an unconventional capacitive sensor based on composite foam materials filled with conductive carbon black particles. Owing to the elastic buckling of the foam pores, the sensitivity exceeds $35 \mathrm{kPa}^{-1}$ for pressure $<0.2 \mathrm{kPa}$. These performances are one order of magnitude higher than the ones previously reported. These materials are low-cost, easy to prepare, and display high capacitance values, which are easy to measure using low-cost electronics. These materials pave the road for the implementation of e-skin in commercialized applications.

npj Flexible Electronics (2019)3:7; https://doi.org/10.1038/s41528-019-0052-6

\section{INTRODUCTION}

The skin is the largest organ of the human body, allowing humans to explore their environment through touch. The imitation of human skin's sensory ability via electronics (e-skin) is raising great interest due to its potential for applications, including humanrobot interactions, prosthesis arms with lifelike sense of touch, drivers' drowsiness detector, and heart monitoring. ${ }^{1-4}$ Recent piezo-resistive materials that emulate tactile sensing via flexible pressure sensors have been reported. ${ }^{5}$ Under pressure, changes in material's electrical resistance are easily achievable by breaking and reforming percolating pathways. Yet, the poor reliability and the high-power consumption of piezo-resistive sensors encourage the scientific community to develop alternatives like piezocapacitive sensors. The most sensitive to pressure piezo-resistive sensors, developed in ref. ${ }^{6}$, consume $>0.1 \mathrm{~mW} / \mathrm{cm}^{2}$. Mannsfeld et al. ${ }^{7}$ microstructured a polydimethylsiloxane (PDMS) film to subtly change the permittivity of piezo-capacitive sensors. They reported a sensitivity of $0.55 \mathrm{kPa}^{-1}$ compared to $0.02 \mathrm{kPa}^{-1}$ for an unstructured PDMS film. However, PDMS has a low permittivity, leading to low recorded capacitive signals. It makes the use of electronic amplifications, like OFET, essential to detect very small pressure changes. Integration of OFET into real life applications is limited by their need of a huge source drain/gate voltage ( $200 \mathrm{~V})$ to work. In addition, surface microtexturing can be degraded with time as a result of friction, wear, and soiling. Actually, the conception of sensitive flexible sensors is conditioned by the possibility to develop new materials.

Here, we present an unconventional composite material, especially designed to combine mechanical robustness, simple fabrication, low energy consumption and unprecedented sensitivity (see Fig. 1a). We use solid foams to enhance the response to mechanical pressure. To the best of our knowledge, this concept is new and differs from the pyramidal microstructures used in refs. ${ }^{7-10}$ In compression, solid foams show linear elasticity at low stresses followed by a long collapse plateau truncated by a regime of densification in which the stress rises steeply. ${ }^{11}$ The plateau is associated to elastic buckling in elastomeric foams. The stress value of the plateau might be tuned by decreasing the Young's modulus of the polymer or by increasing the amount of air in the foam. On this plateau, a slight variation of pressure induces a large variation of deformation. In the following, we take advantage of this feature. We design solid foams whose plateau stress value corresponds to the range of pressure we plan to measure. We use electrostrictive materials to convert the strain deformation into a huge variation of capacitance. For this purpose, we prepare a PDMS porous materials of closed porosity by using an emulsion road. The surface of the closed pores is covered with carbon black (CB) conductive particles. These particles increase the dielectric constant of the composites by enhancing the interfacial polarization (Maxwell-Wagner effect) with the creation of local microcapacitor. As the micropores and, thus, the micro-capacitors significantly deform upon compression, we achieve a large change of the permittivity associated to large changes in capacitance. The materials are prepared with the highest concentration of carbon black particles and with the highest amount of dispersed phase in order to reach the best performances in terms of sensitivity. However, the large increase of electrostriction is also accompanied by an increase of conductivity, which is detrimental for energy consumption. To circumvent this weakness, a thin insulating layer is placed at the bottom of the composite. The thickness of this layer is chosen in order to keep a high effective capacitance of the bilayer structure. With these bilayer composites, we demonstrate the large fabrication of flexible capacitive pressure sensors with a high

\footnotetext{
${ }^{1}$ Chimie Biologie Innovation, ESPCI Paris, CNRS, PSL University, 75005 Paris, France; ${ }^{2}$ Sciences et Ingénierie de la Matière Molle, ESPCI Paris, CNRS, PSL University, 75005 Paris, France and ${ }^{3}$ Centre de Recherche Paul Pascal, CNRS, Université de Bordeaux, 115 Avenue Schweitzer, 33600 Pessac, France

Correspondence: Philippe Poulin (poulin@crpp-bordeaux.cnrs.fr) or Annie Colin (annie.colin@espci.fr)
}

Received: 13 December 2018 Accepted: 20 March 2019

Published online: 04 April 2019 
A

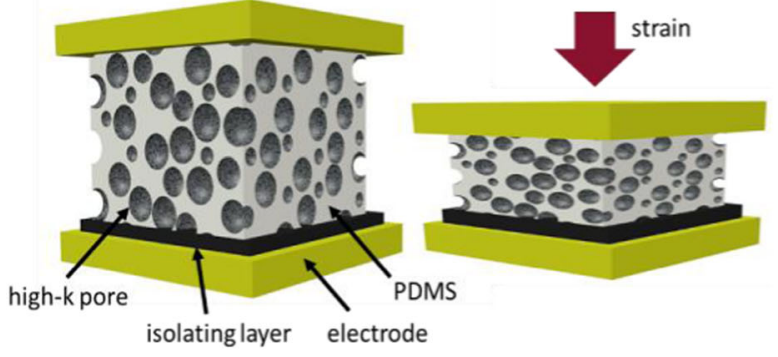

B

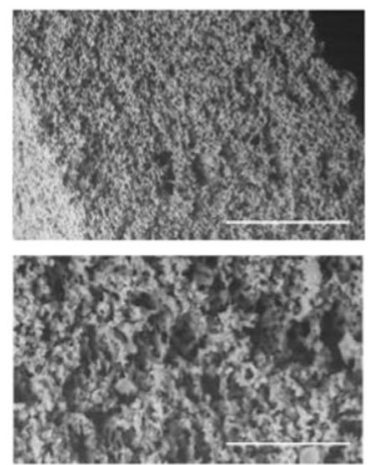

C

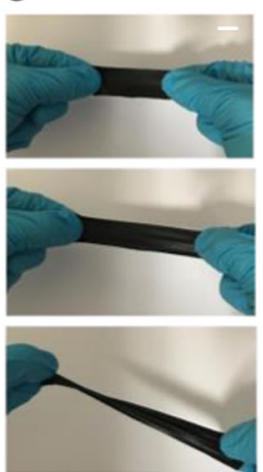

Fig. 1 a Schematic view of polymeric foam filled with conductive pores. b Slice views of the internal structure of the CB/PDMS porous material (SEM, scanning electronic microscope), scale bars: top, $300 \mu \mathrm{m}$ and bottom, $100 \mu \mathrm{m}$. c From top to bottom: CB/PDMS porous material at rest, stretched and twisted, scale bar, $1 \mathrm{~cm}$

sensitivity ( $>35 \mathrm{kPa}^{-1}$ ) without use of any electronic amplification. The sensitivity is at least 70 times greater than previously reported values. Moreover, our sensor consumes $<220 \mathrm{pW} / \mathrm{cm}^{2}$. It involves capacitance values higher than $100 \mathrm{pF}$, i.e., easily measurable with low-cost electronics. We show the performances of these bilayer materials by continuously recording arterial pulse waves in a noninvasive and nonintrusive way.

\section{RESULTS}

We fabricate the CB/PDMS piezo-capacitive layer using a water-inoil (W/O) emulsion method. The emulsion consists of an aqueous solution of carbon black droplets dispersed in a matrix of PDMS and a curing agent. The oil phase is prepared by mixing PDMS (RTV615 Momentive), the curing agent (RTV615 Momentive, 10\% in weight with respect to the PDMS phase), and the surfactant lauryl PEG-8 dimethicone (Silube J208-812, Siltech, $5.0 \mathrm{wt} \%$ of the final mixture). Note that the choice of these compound is fundamental if one wants to obtain a low Young modulus. The water phase is prepared by mixing $5 \mathrm{~g}$ of Arabic gum (Sigma Aldrich) and $95 \mathrm{~g}$ of deionized water. The desired concentration of carbon black powder (Alfa Aesar) is added and the mixture is tip sonicated for $1 \mathrm{~h}$ at $400 \mathrm{~W}$ to homogeneously disperse the carbon black particles. The emulsion is prepared by progressively adding the carbon black solution to the oil-phase under manual stirring. The water-in-oil emulsion loaded with carbon black particles is spread with a stencil that has a depth of $900 \mu \mathrm{m}$ on a plastic surface with a diameter of $24 \mathrm{~mm}$. A second plastic surface is placed on the spread emulsion such that the emulsion remains confined between two flat surfaces. Solid materials are obtained by curing the PDMS polymer without evaporation in a warm water bowl $\left(90^{\circ} \mathrm{C}\right)$ for $4 \mathrm{~h}$. The relative humidity in these conditions is $100 \%$. Then, the solid material layer is removed from the two plastic surfaces and dried in an oven for $1 \mathrm{~h}$ at $150^{\circ} \mathrm{C}$. As PDMS is permeable to water vapor, droplets from carbon black solution dried and leave a structure with spherical pores covered by carbon black particles as previously described in ref. ${ }^{12}$ (see Materials and methods). After water removal and PDMS cross-linking, this procedure leads to porous PDMS with a low Young's modulus compared to bulk PDMS.

We systematically vary the concentrations of carbon black, dispersed-phase concentration, and insulating film thickness in order to obtain a suitable material for a pressure sensor (see Fig. S1, Supporting information). First, we measure the dielectric properties at $1 \mathrm{kHz}$ as a function of the carbon black concentration. We determine the optimal concentration to combine a high dielectric constant and a low conductivity. A frequency of $1 \mathrm{kHz}$ is chosen in order to correctly sample a cardiac pulse signal (see Fig. 4c). As promising materials for pressure sensing, we select samples whose relative dielectric constant is above 30 and whose loss tangent is $<0.2$.

Second, the porosity is varied by changing the dispersed fraction of the emulsions. The cross-linking agent that we use (Momentive RTV615) allows to make stable emulsions up to a dispersed-phase fraction of $80 \mathrm{wt} \%$. This large volume fraction allows a very low Young's modulus (below $0.15 \mathrm{MPa}$ ). Related materials with lower volume fractions of carbon suspensions were found to be much stiffer. ${ }^{12}$ Hence, the optimal dispersed-phase fraction is $80 \mathrm{wt} \%$.

Since the conductivity of the majority of the samples remains high, we add an insulating dielectric layer to reduce the conductivity of the all device. The optimal thickness of $2.5 \mu \mathrm{m}$ has been obtained using a simple modeling of resistance in series (see Fig. S2, Supporting information). A thin plastic film of polyethylene terephthalate (PET, thickness of $2.5 \mu \mathrm{m}$ ) is directly stacked on the solid CB/PDMS composite by using a surface plasma treatment for $3 \mathrm{~min}$ and by applying a pressure at $90^{\circ} \mathrm{C}$ between the two pieces (see Materials and methods).

For the pressure sensor applications, three composites are studied with 0,3 , and 10 wt $\%$ of $C B$, respectively, regarding the PDMS (thickness $1.2 \mathrm{~mm}$ ) from $\mathrm{W} / \mathrm{O}$ emulsions concentrated at $80 \mathrm{wt} \%$. Above $10 \mathrm{wt} \%$ of $\mathrm{CB}$, a poor homogenization of the carbon particles was observed. This is commented in supporting information (see Note S1, Supporting information).

The thickness of the samples is set by the distance between the two plastic surfaces used during the material preparation. Figure $1 \mathrm{~b}$ shows the internal structure of a $10 \mathrm{wt} \% \mathrm{CB}$ composite where the porosity is clearly visible. The high flexibility and the fabrication on large scale of the composite are illustrated in Fig. 1c in which we show the material submitted to stretch and twist.

We operate the bilayer materials (CB/PDMS + PET) in a capacitor configuration (Fig. 2a) while applying linear loading-unloading cycles (see Materials and methods). We load the sample between two electrodes and we record the normal force, the displacement, the capacitance $C$, and the resistance $R$. The displacement $d$ corresponds to the gap between the top and the bottom electrode. The zero position i.e., $d=0 \mathrm{~mm}$ corresponds to the contact between the two electrodes in absence of sample. The zero stress corresponds to the first $d$ point i.e., $d=1.3 \mathrm{~mm}$ see Fig. 2b. We defined the pressure as the normal force divided by the area at the start of the experiment $\left(A_{0}\right)$, this is the engineering stress ( $\neq$ true stress, i.e., the change of area upon compression is neglected).

We first study the mechanical behavior of a porous $10 \mathrm{wt} \% \mathrm{CB}$ composite with a PET layer. From the SEM pictures, we measure 
A

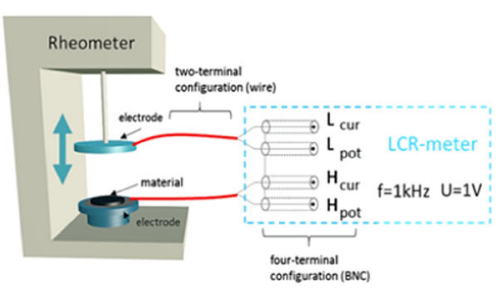

B

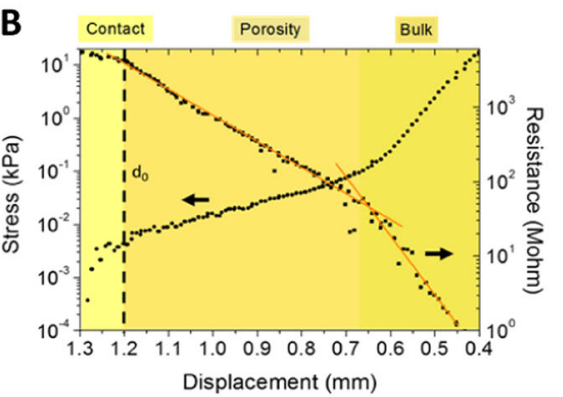

C

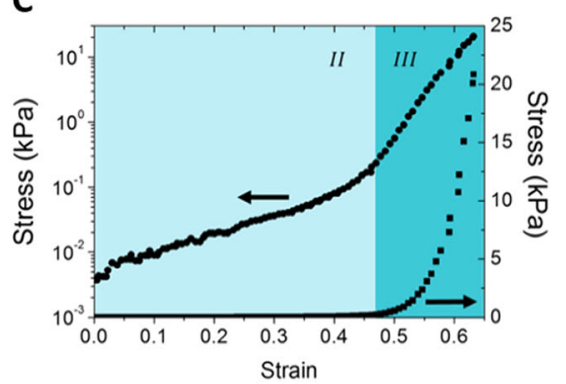

Fig. 2 a Schematic representation of the experimental set-up for sensitivity measurements. The bilayer material is placed between the two stainless electrodes, the bottom electrode is at rest while the top electrode can be moved. Rheometer is used as mechanized $z$-axis stage and force gauge. Dielectric properties are measured using an LCR-meter at $1 \mathrm{kHz}$ and a bias of $1 \mathrm{~V}$. $\mathbf{b}$ Evolution of the stress and the electrical resistance $R$ as function of the displacement, $10 \mathrm{wt} \%$ CB bilayer material. c Stress-strain curve after 100 cycles, 10 wt $\%$ CB bilayer material. Pressure ranges are highlighted by blue increments: blue II $(0-0.2 \mathrm{kPa})$, blue III $(0.2-20 \mathrm{kPa})$

the material thickness $e$ to be $1210 \mu \mathrm{m}$ and estimate its roughness to $40 \mu \mathrm{m}$ (typically three emulsion droplets diameters).

In semi-log scale, the resistance as a function of the distance displays three different slopes. These regimes are evidenced in Fig. $2 \mathrm{~b}$ and are denoted by I, II, and III.

In regime $l$, we measure a very high resistance. This regime corresponds to the regime contact. At $d=1.3 \mathrm{~mm}$, the electrodes brush the material. At $d=d_{0}$, the contact between the material and the electrodes is perfectly established. Regime I corresponds to a thickness of $80 \mu \mathrm{m}$. On both faces of the material, the asperities are measured to be $40 \mu \mathrm{m}$, which is in agreement with the measured size of regime $I$. In this regime, the material can be viewed as a mixture of air and foam. The conductivity of the layer located between 0 and $d_{0}$ is equal to $1.33 \times 10^{-10} \mathrm{~S} / \mathrm{m}$, which is much higher than air in the laboratory conditions $\left(4 \times 10^{-12} \mathrm{~S} / \mathrm{m}\right)$ and much less than uncompressed material $10^{-9} \mathrm{~S} / \mathrm{m}$. This last value is computed assuming a resistance of $4 \times 10^{9} \Omega$ for a thickness of $1.2 \mathrm{~mm}$ and an area of $3 \times 10^{-4} \mathrm{~m}^{2}$.

In regime II, the resistance varies exponentially with the displacement. The decrease of $R$ is, thus, much greater than that expected from simple geometrical deformation (i.e., linear variation). This suggests that the structure of the materials evolves strongly as a function of the compression. Regime III corresponds to the stiffening of the materials and to the closure of some pores.

Figure $2 \mathrm{c}$ shows the compressive stress-strain curves for a porous PDMS $/ 10 \%$ CB material. The strain is defined as $S=\frac{d_{0}-d}{d_{0}}$. We evidence two mechanical behaviors: a slowly increase in stress from 0 to $0.2 \mathrm{kPa}$ when the strain increases from 0 to 0.47 (regime II) followed by a faster increase in stress above a strain of 0.47 (regime III). Regime II is associated to a deformation of the pores by elastic buckling followed by a closing of the pores in regime III. ${ }^{11}$ The closing of the pores is reflected by a decrease in the slope of the resistance. When the $C B$ pores are almost totally closed, a further strain compresses the PDMS itself resulting into a Young's modulus of $0.15 \mathrm{MPa}$. The Young's modulus is defined here as the local slope of stress-strain curve. Stress-strain curves have been measured for different loading-unloading consecutive cycles and showed very low hysteresis after 100 cycles (Fig. S3, Supporting information).

Figure 3 a shows the capacitance for three materials $(0,3,10 \mathrm{wt} \%$ CB) with a diameter of $20 \mathrm{~mm}$ and a thickness of $1.2 \mathrm{~mm}$ depending on the applied pressure. We find that increasing the carbon concentration leads to a clear enhancement of the capacitance: Under $1 \mathrm{kPa}$, the material without carbon has only a capacitance of $20 \mathrm{pF}$, whereas the $3 \mathrm{wt} \% \mathrm{CB}$ material displays a capacitance of $100 \mathrm{pF}$. Moreover, the $10 \mathrm{wt} \% \mathrm{CB}$ material has a capacitance of $170 \mathrm{pF}$ under equal pressure, i.e., $70 \%$ higher than that of the $3 \mathrm{wt} \%$ CB material (see Fig. S9, Supporting information, for the frequency dependence of the capacitance). These large values of capacitance allow electronic signals to be easily measured and make the use of an electrical amplifier such as an OFET superfluous. In classical conditions, a large concentration of carbon black would lead to high electrical conductivity. ${ }^{12}$ However, a resistance of $10 \mathrm{M} \Omega$ at $1 \mathrm{kPa}$ is measured for the $10 \mathrm{wt} \%$ CB (see Fig. S4, Supporting information), which leads to a power consumption of about $100 \mathrm{nW}$ for a bias of $1 \mathrm{~V}$ (see Fig. S5, Supporting information). To compare, a conventional silicon piezoresistive pressure transducer has a typical consumption of several $\mathrm{mW}$ through resistive Joule's heating. ${ }^{13}$

Next, we study the cyclability of the porous bilayer materials. The performance of the $10 \mathrm{wt} \% \mathrm{CB}$ material over consecutive loading-unloading cycles is shown in Fig. 3b. The consecutive cycles demonstrate little changes in the pressure-response curves. The inset shows that the sensitivity remains quasi-constant over the consecutive cycles (see Fig. S6, Supporting information for 1000 cycles). The bilayer material exhibits no hysteresis. Figure $3 \mathrm{c}$ exhibits the different sensitivities of the $10 \mathrm{wt} \%$ CB bilayer material depending on the pressure ranges. The maximum slope of the relative capacitive change is measured in the $0-0.2 \mathrm{kPa}$ range with a value of $35.1 \mathrm{kPa}^{-1}$, corresponding to the pore-closing regime (regime II). Then values of $6.6 \mathrm{kPa}^{-1}$ and $3.5 \mathrm{kPa}^{-1}$ are, respectively, measured in the $0.2-1.5 \mathrm{kPa}$, and $1.5-0 \mathrm{kPa}$ range, corresponding to the bulk regime (regime III). Until now, the best reported sensitivity with no amplification and a microstructured PDMS film has been reported by Mannsfeld et al. $\left(0.55 \mathrm{kPa}^{-1}\right){ }^{7}$ The present porous bilayer material display a much greater sensitivity. Moreover, the performances of our material are higher than the best sensitivity achieved with amplification $\left(8.2 \mathrm{kPa}^{-1}\right)$, reached by Schwartz et al. ${ }^{14}$ with an OFET configuration and a pyramid microstructuration. These performances are accompanied by a signal to noise ratio (SNR) of $4 \times 10^{5}$ for a pressure as low as 8.8 Pa (see Fig. S7, Supporting information). This is an additional advantage above the high sensitivity, which will make the final sensor conditioning easier. Also we can conclude that the limit of detection is below $8.8 \mathrm{~Pa}$. We cannot determine the exact limit because it is limited in our experiments by the force sensor resolution (see Note S2, Supporting information).

Beyond the high sensitivities to pressure, our material shows also high capacitive gauge factor in its bulk regime (regime III). Figure $3 \mathrm{~d}$ shows the capacitance variation as a function of the strain for a mechanical compression. We remind that the gauge factor can be defined as the slope of the capacitance variation vs strain curves. The sensor device shows reliable and linear performance over thousands of cycles at up to $10 \%$ strain, and the highest reported gauge factor for a device of this class: $31.7 .^{5}$ The theoretical value of gauge factor for incompressible materials due to geometrical change and assuming a constant dielectric constant is 1 (refs. ${ }^{15,16}$ ). As our materials have shown excellent electrostrictive properties (permittivity variation under compression), we succeed in increasing the gauge factor by a factor 30 . 

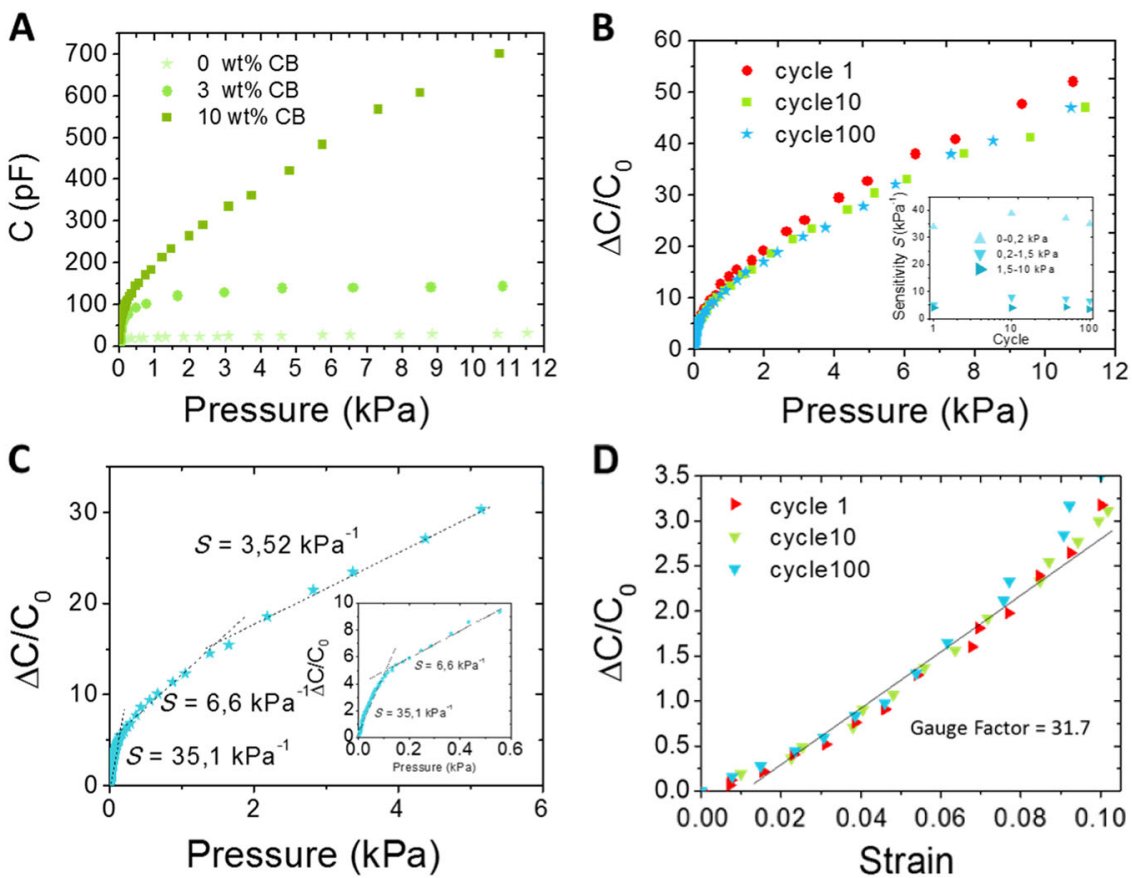

Fig. 3 a Capacitances as a function of the applied pressure for three bilayer materials: $0 \%$ CB (stars); $3 \%$ CB (circles); $10 \%$ CB (squares). b Pressure-response curves for successive loading-unloading cycles of the $10 \mathrm{wt} \%$ CB material: 1st cycle (circles), 10th cycle (square), and 100th cycle (stars). Inset: sensitivity $S$ for the three different pressure ranges as a function of cycle number. c Sensitivity measurements $S$ after 100 cycles for three different pressure ranges (10 wt\% CB). d Capacitance variations as function of the strain in the bulk domain (Zone III) for successive loading-unloading cycles (red triangles for one cycle, green triangles for ten cycles, and blue triangles for 100 cycles)

A

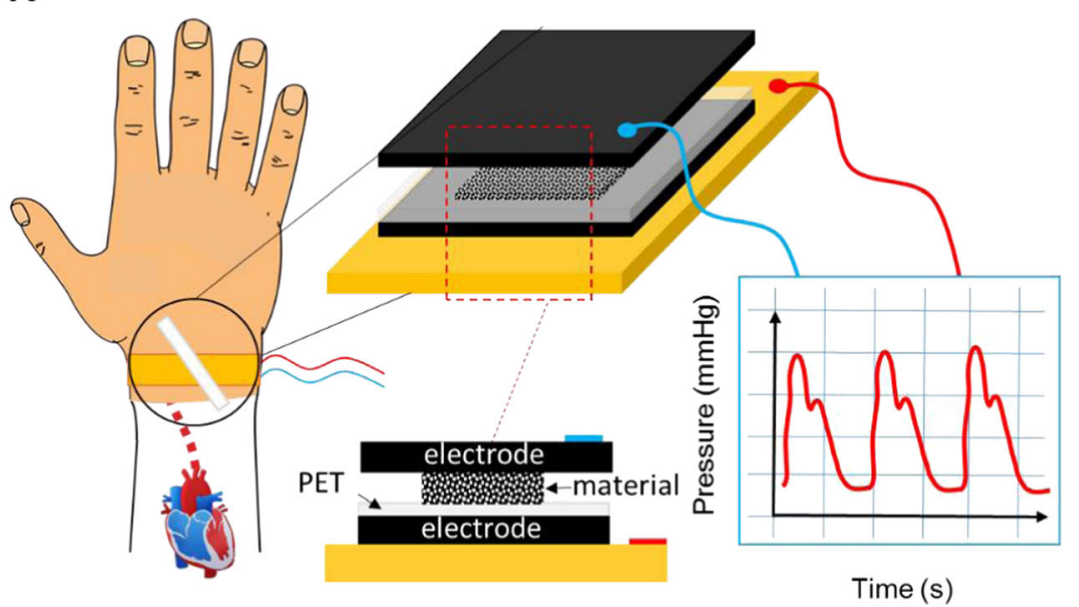

B
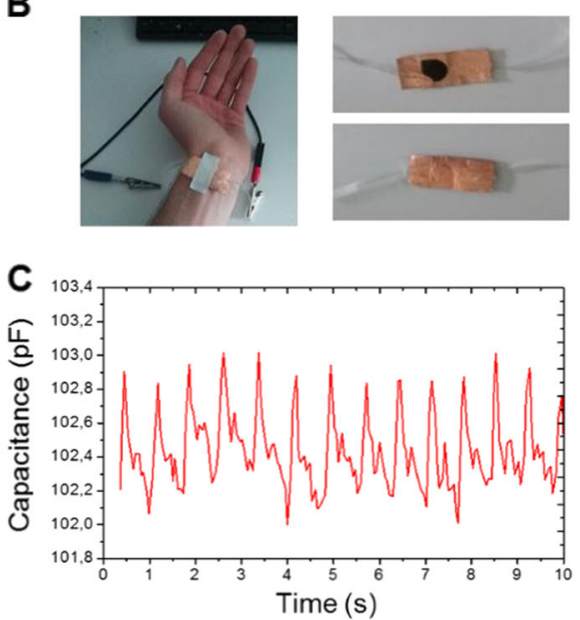

Fig. 4 a Schematic illustration of the capacitive pressure sensor. $\mathbf{b}$ The pressure sensor attached to the wrist of a person. $\mathbf{c}$ Real-time and insitu capacitive signal of the artery wrist pulse recorded at $1 \mathrm{kHz}$ under application of $1 \mathrm{~V}$ using an LCR-meter (see Note S3 Supporting Information for voltage choice). The recorded wave signal corresponds to the typical human waveform

Additionally, we tried to estimate the response of time of the material to pressure variations by loaded/unloaded it (13.2 Pa or $9 \mathrm{~Pa}$ ) with a piezoelectric actuator $(0.1 \mathrm{~Hz}$ square signal). This time is below the time resolution of our LCR-meter $(80 \mathrm{~ms})$ (see Fig. S8, Supporting information)

In the medical field, the arterial pulse wave is a key indicator as it is intimately related to the physical properties of the cardiovascular system. To illustrate the capabilities of the highly sensitive present pressure sensor, we place the sensor over the radial artery of the wrist to measure pulse waves. This is a standard test. ${ }^{14,17}$ The sensitive material was sandwiched between two compliant electrodes by depositing conductive flexible carbon tape on the top and the bottom of the materials surfaces as illustrated in Fig. 4a. The system electrode-material-electrode is deposited on a copper tape, used as a substrate. The device, connected by electric wires, is simply deposited on the wrist and fixed with non-conductive tape (Fig. 4b). It is worth mentioning that contrary to usual techniques no compressive cuff is required. This advantage makes our device suited for non-invasive and continuous blood-pressure monitoring. As low-pressure capacitive sensors do not require cuff, but just to be left on the wrist, a conversion of the capacitive signal (pulse wave) into a pressure value has to be operated. The use of machine learning algorithms is the current approach to get cuffless bloodpressure estimation from a pulse wave with enough accuracy. Progress in this direction has been recently reported. ${ }^{18}$ We believe that combined with our sensitive materials the use of these 

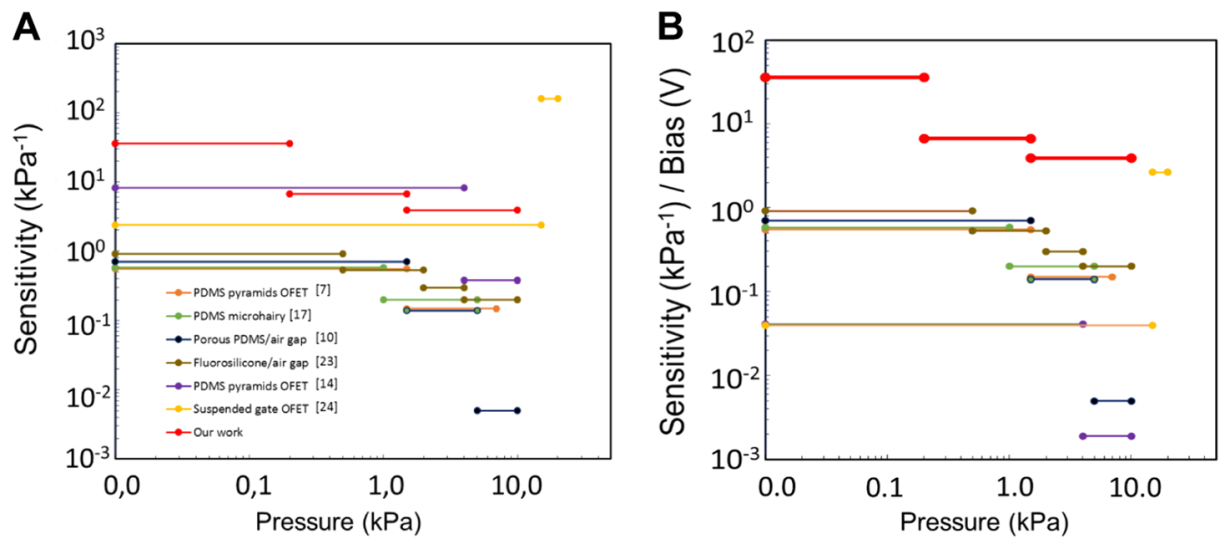

Fig. 5 a Comparison of the sensitivity of capacitive pressure sensors as a function of pressure. The plot includes the values reported from refs. ${ }^{7,10,14,17,23,24}$ and from this work. $\mathbf{b}$ Sensitivity over the used bias for the same systems as in $\mathbf{a}$

algorithms could lead to cuffless devices to monitor the bloodpressure which would useful both from the clinical and practical perspectives. The fast response time of the sensor provides high resolution, which aims at getting the detailed clinical information from wrist pulse wave. For healthy humans, the systolic pressure (maximum during one heart beat) is $90-120 \mathrm{~mm} \mathrm{Hg}^{19}(10.5-16 \mathrm{kPa})$ and the diastolic pressure (minimum in between two heart beats) is $60-79 \mathrm{~mm} \mathrm{Hg}^{19}$ (8-10.5 $\mathrm{kPa}$ ). The arterial pressure range of healthy people is $8-16 \mathrm{kPa}$. However, as a skin layer remains above the radial artery, the accessible pressure on the skin surface is much lower. Figure $4 c$ shows three wave pulses of a healthy young man recorded by the pressure sensor. The waveform is regular and repeatable at 75 beats per minute. For measuring the bloodpressure, a calibration between the measured capacitance and the pressure needs to be precisely established. The recorded signal shows that blood-pressure is fully measurable and the typical waveform (systolic and diastolic peaks) of the arterial pulse is clearly and accurately defined.

\section{DISCUSSION}

In conclusion, we developed a supersensitive capacitive pressure sensor by combining porous CB/PDMS composites with a thin PET isolating layer. The association of a porous material filled with conductive particles addresses the mechanical (low Young's modulus) and dielectric requirements (high- $\kappa$ ) for developing flexible dielectric layers. The material shows unprecedented properties affording sensitivities of $35.1 \mathrm{kPa}^{-1}$ in the $0-0.2 \mathrm{kPa}$ pressure range and $6.6 \mathrm{kPa}^{-1}$ in the $0.2-1.5 \mathrm{kPa}$ range. These performances are one order of magnitude higher than those recently reported (Fig. 5a). The high capacitance ( $>100 \mathrm{pF})$ are easy to measure with low-cost electronics. Until now, capacitive pressure sensors based on rubber were mostly obtained using very delicate patterning process (pyramid-structured PDMS). Moreover, they achieved high sensitivities by operating them with thin-film transistors (OFET) for signal amplification. A bias of only $1 \mathrm{~V}$ has been used to record our capacitive responses. The comparison of sensitivities over the used bias (Fig. 5b) places our sensors well-above the previous reported works. Furthermore, our sensor performs stable pressure-capacitance changes and sensitivities after hundreds of cycles. The sensor has been successfully employed to assess the pulse frequency and to measure systolic and diastolic peaks of an arterial pulse wave. This provides a solution to the dilemma of combining non-invasive and continuous blood-pressure monitoring. This bilayer material also offers opportunities to make highly sensitive and wearable capacitive sensors in other fields at low-cost and low-power consumption electronic systems. Indeed, although the pressure threshold of a human finger is about $10 \mathrm{kPa}^{20}$ the high-pressure sensitivity of our material could pave the way of artificial skin rivaling with human skin. In this context, robotic palpation from soft tissue organs is an important field of research. ${ }^{21}$ For example, the detection of embedded nodules in soft tissues requires highly sensitive sensors as the variation of stiffness is below $0.1 \mathrm{kPa}$ for very small nodules $(\mathrm{mm}){ }^{22}$

\section{METHODS}

Material synthesis

The emulsion consists of droplets of an aqueous dispersion of carbon black particles suspended in a matrix of PDMS and a curing agent. The oil-phase is prepared by mixing PDMS (RTV615 Momentive), the curing agent (RTV615 Momentive, 10\% in weight with respect to the PDMS phase), and the surfactant lauryl PEG-8 dimethicone (Silube J208-812, Siltech, 5.0 wt\% of the final mixture). The water phase is prepared by mixing $5 \mathrm{~g}$ of Arabic gum (Sigma Aldrich) and $95 \mathrm{~g}$ of deionized water. The desired amount of carbon black powder (Alfa Aesar) is added and the mixture is tip sonicated for $1 \mathrm{~h}$ at $400 \mathrm{~W}$ to homogeneously disperse the carbon black (CB) particles. The emulsion is prepared by progressively adding the carbon black solution to the oil phase under manual stirring up to a mass ratio water:oil of $80 \% \mathrm{wt}$. The water-in-oil emulsion loaded with carbon black particles is spread with a stencil that has a depth of $1.2 \mathrm{~mm}$ on a plastic surface with a diameter of $24 \mathrm{~mm}$. A second plastic surface is placed on the spread emulsion such that the emulsion remains confined between two flat surfaces. Solid materials are obtained by curing the PDMS polymer without evaporation in a warm water bowl $\left(90^{\circ} \mathrm{C}\right)$ for $4 \mathrm{~h}$. The relative humidity in these conditions is $100 \%$. Then, the solid material layer is removed from the two plastic surfaces and dried in an oven for $1 \mathrm{~h}$ at $150^{\circ} \mathrm{C}$. As PDMS is permeable to water vapor, droplets from carbon black solution dried and leave a structure with spherical-shaped pores covered by carbon black particles.

Insulating layer deposition

A PET film $(2.5 \mu \mathrm{m}$, Radiospare) is stacked on the solid CB/PDMS composites by using a surface plasma treatment (Plasma Diener Pico). Both surfaces are treated for $3 \mathrm{~min}$. This method aims at making sticky, the thin plastic film, on PDMS composites. Then the two elements are stuck together for $15 \mathrm{~min}$ by applying a pressure at $90^{\circ} \mathrm{C}$ in an oven.

\section{Material characterization}

The sensitivity to compression tests of the bilayer composites are measured using the experimental configuration depicted in Fig. 2a. To apply a well-controlled load, a rheometer is used as mechanized $z$-axis stage and force gauge. A top electrode (stainless steel) is connected to the $z$-axis stage while the bottom electrode is fixed. A Discovery HR-2 (TA Instruments) applies linear loading-unloading cycles to the sensing materials. At the same time the dielectric properties are measured at $1 \mathrm{kHz}$ with a bias of $1 \mathrm{~V}$ using an LCR-meter (Agilent). To do so, the material is tested into a four measurement ports circuit (Lcur, Lpot, Hcur and Hpot, see Fig. 2a). An AC voltage is first supplying out of the high-current terminal (Hcur). Then, the current through the tested material is measured 
by the low current terminal (Lcur), and the voltage across the tested material is measured by the high- and low- potential terminals (Hpot and Lpot). The internal correction functions (cable length correction, open and short corrections) of the LCR-meter are used to correct errors due to the test fixture and the test leads. To perform the open correction, BNC and wires are connected to the electrodes and LCR-meter. A $5 \mathrm{~mm}$ air gap is maintained between the electrodes. The short-circuit correction is made by shorting the two electrodes. The force, the displacement, the capacitance and the resistance are recorded through an interfaced computer. The size of the pressure-sensitive material is $3.1 \mathrm{~cm}^{2}$.

\section{Pressure sensor fabrication}

To fabricate the pressure sensor, the sensitive material was completed by two flexible electrodes by depositing conductive flexible carbon tape (Neyco) on the top and the bottom of the materials surfaces. The system electrode-material-electrode is deposited on a copper tape (Radiospare), used as a substrate. The device, completed by wires, is simply deposited on the wrist and fixed with non-conductive tape.

\section{DATA AVAILABILITY}

The data that support the findings of this study are available from the corresponding author, A.C. or P.P., upon reasonable request.

\section{ACKNOWLEDGEMENTS}

This research is supported by the Agence Nationale de la Recherche (projet POETICS). M.P. thanks DIM poreux for a financial support.

\section{AUTHOR CONTRIBUTIONS}

M.P. and W.S. fabricated the materials and conducted the characterization. P.P, C.M., and A.C. carried out experimental design. M.P, W.S., and A.C. performed data analysis. M.P., W.S., P.P., and A.C. wrote the paper. All authors discussed the results and commented on the manuscript.

\section{ADDITIONAL INFORMATION}

Supplementary information accompanies the paper on the npj Flexible Electronics website (https://doi.org/10.1038/s41528-019-0052-6).

Competing interests: The authors declare no competing interests.

Publisher's note: Springer Nature remains neutral with regard to jurisdictional claims in published maps and institutional affiliations.

\section{REFERENCES}

1. Wang, X. et al. Recent progress in electronic skin. Adv. Sci. 2, 1500169 (2015).

2. Kim, Y. et al. A bioinspired flexible organic artificial afferent nerve. Science $\mathbf{3 6 0}$, 998-1003 (2018).

3. Zeng, W. et al. Fiber-based wearable electronics: A review of materials, fabrication, devices, and applications. Adv. Mater. 26, 5310-5336 (2014).

4. Yan, W. et al. Advanced multimaterial electronic and optoelectronic fibers and textiles. Adv. Mater. 31, 1802348 (2019).

5. Trung, T. Q. \& Lee, N. -E. Flexible and stretchable physical sensor integrated platforms for wearable human-activity monitoring and personal healthcare. Adv. Mater. 28, 4338-4372 (2016).

6. $\mathrm{Hu}, \mathrm{L}$ et al. Piezoresistive behavior of porous carbon nanotube-thermoplastic polyurethane conductive nanocomposites with ultrahigh compressibility. Appl. Phys. Lett. 108, 011904 (2016).
7. Mannsfeld, S. C. B. et al. Highly sensitive flexible pressure sensors with microstructured rubber dielectric layers. Nat. Mater. 9, 859-864 (2010).

8. Boutry, C. M. et al. A sensitive and biodegradable pressure sensor array for cardiovascular monitoring. Adv. Mater. 27, 6954-6961 (2015).

9. Wang, X., Gu, Y., Xiong, Z., Cui, Z. \& Zhang, T. Silk-molded flexible, ultrasensitive, and highly stable electronic skin for monitoring human physiological signals. Adv. Mater. 26, 1336-1342 (2014).

10. Park, S. et al. Stretchable energy-harvesting tactile electronic skin capable of differentiating multiple mechanical stimuli modes. Adv. Mater. 26, 7324-7332 (2014).

11. Gibson, L. J. \& Ashby, M. F. Cellular Solids: Structure and Properties. (Cambridge University Press, 1997).

12. Pruvost, M., Smit, W. J., Monteux, C., Poulin, P. \& Colin, A. Microporous electrostrictive materials for vibrational energy harvesting. Multifunct. Mater. 1, 015004 (2018).

13. Pavlin, M., Belavic, D. \& Novak, F. Ceramic MEMS designed for wireless pressure monitoring in the industrial environment. Sensors 12, 320-333 (2011).

14. Schwartz, G. et al. Flexible polymer transistors with high pressure sensitivity for application in electronic skin and health monitoring. Nat. Commun. 4, 1859 (2013).

15. Cohen, D. J., Mitra, D., Peterson, K. \& Maharbiz, M. M. A highly elastic, capacitive strain gauge based on percolating nanotube networks. Nano Lett. 12, 1821-1825 (2012).

16. Yao, S. \& Zhu, Y. Wearable multifunctional sensors using printed stretchable conductors made of silver nanowires. Nanoscale 6, 2345 (2014).

17. Pang, C. et al. Highly skin-conformal microhairy sensor for pulse signal amplification. Adv. Mater. 27, 634-640 (2015).

18. Khalid, S. G., Zhang, J., Chen, F. \& Zheng, D. Blood pressure estimation using photoplethysmography only: Comparison between different machine learning approaches. J. Healthc. Eng. 1-13 (2018).

19. Whelton, P. K. et al. Guideline for the prevention, detection, evaluation, and management of high blood pressure in adults: A report of the American College of Cardiology/American Heart Association Task Force on Clinical Practice Guidelines. Hypertension 71, e13-e115 (2018).

20. Dellon, E. S., Mourey, R., Dellon, A. L. \& Dellon, A. L. Human pressure perception values for constant and moving one- and two-point discrimination. Plast Reconstr. Surg. 90, 112-117 (1992).

21. Okamura, A. M. Haptic feedback in robot-assisted minimally invasive surgery. Curr. Opin. Urol. 19, 102-107 (2009).

22. Konstantinova, J., Cotugno, G., Dasgupta, P., Althoefer, K. \& Nanayakkara, T. Autonomous robotic palpation of soft tissue using the modulation of applied force. in 2016 6th IEEE International Conference on Biomedical Robotics and Biomechatronics (BioRob) 323-328 (IEEE, 2016). https://doi.org/10.1109/BIOROB.2016.7523646.

23. Viry, L. et al. Flexible three-axial force sensor for soft and highly sensitive artificial touch. Adv. Mater. 26, 2659-2664 (2014).

24. Zang, Y. et al. Flexible suspended gate organic thin-film transistors for ultrasensitive pressure detection. Nat. Commun. 6, (2015). Attribution 4.0 International License, which permits use, sharing, adaptation, distribution and reproduction in any medium or format, as long as you give appropriate credit to the original author(s) and the source, provide a link to the Creative Commons license, and indicate if changes were made. The images or other third party material in this article are included in the article's Creative Commons license, unless indicated otherwise in a credit line to the material. If material is not included in the article's Creative Commons license and your intended use is not permitted by statutory regulation or exceeds the permitted use, you will need to obtain permission directly from the copyright holder. To view a copy of this license, visit http://creativecommons. org/licenses/by/4.0/.

(c) The Author(s) 2019 